\title{
AN OPEN LABEL COMPARISON OF CHLORDIAZEPOXIDE AND LORAZEPAM IN ALCOHOL WITHDRAWAL
}

Rajmohan $\mathrm{V}^{1}$.

1. Assistant Professor, Department of Psychiatry, MES Medical College, Perintalmanna.

\section{CORRESPONDING AUTHOR}

Dr. Rajmohan V,

Apartment 2A.

Kingswood Apartment,

Bilathikulam, Kozhikode Pin: 673006.

Email- rajgiggsmohan@yahoo.com

\section{HOW TO CITE THIS ARTICLE:}

Rajmohan V. "An Open Label Comparison of Chlordiazepoxide and Lorazepam in Alcohol Withdrawal". Journal of Evolution of Medical and Dental Sciences 2013; Vol2, Issue 25, June 24; Page: 4609-4613.

ABSTRACT:- AIM: To compare the efficacy of lorazepam and chlordiazepoxide in alcohol withdrawal in an open label design. METHOD: The study sample consisted of 103 consecutive admissions to the de addiction ward, which after informed consent and selection based on inclusion criteria were randomised to two groups. A prospective inpatient analysis of withdrawal was done using CIWA-Ar and a comparison of the efficacy of chlordiazepoxide and lorazepam was done post randomization. Measurements the initial withdrawal assessment and subsequent progress of withdrawal was assessed using CIWA-Ar. The rate of withdrawal and total duration of withdrawal was compared among the two groups. RESULTS: Lorazepam treated group showed a significant difference in the rate of improvement over 48 hours compared to chlordiazepoxide group (71.3\% vs. $57.4 \% ; \mathrm{p}=0.000)$. The total duration of withdrawal was also significantly lesser with lorazepam (5.7 vs. 6.9 days; $p=0.001$ ). A stepwise linear regression showed that $63 \%$ of the variance in improvement was related to the drug, thus proving the superiority of lorazepam. CONCLUSION: Lorazepam is more effective than chlordiazepoxide in alcohol withdrawal in both faster rate of fall of withdrawal severity and bringing down the total duration of withdrawal

KEYWORDS: Dependence, Withdrawal, CIWA-Ar, Lorazepam, Chlordiazepoxide

Alcohol withdrawal affects people accustomed to regular alcohol intake, who either decrease consumption or stop drinking completely. The symptoms range from minor ones such as insomnia and tremulousness to severe complications such as withdrawal seizures and delirium tremens. ${ }^{1}$ The role of benzodiazepines though recognised, mean benzodiazepine dosage requirements in patients hospitalized for alcohol withdrawal appear higher than previously reported. ${ }^{2}$ The study attempts to conduct an open label comparison of the efficacy of chlordiazepoxide and lorazepam in the treatment of withdrawal.

METHOD SAMPLE: The study was conducted on an inpatient basis at the de addiction ward of the Department of Psychiatry Medical College, Calicut from January to November, 2005. The sample was 


\section{ORIGINAL ARTICLE}

collected from consecutive patients admitted to the de-addiction ward. The patients were included in the study based on the basis of the following criteria :(a) willingness to participate in the study after full informed consent (b) meeting the criteria for dependence as per ICD-10 (DCR) (c) currently having withdrawal symptoms and having a CIWA-Ar score of 8 or greater at the time of admission. Patients were excluded from the study based on the following (a) patients who were abstinent and treated with counter tolerant drugs just, prior to admission (b) patients' being treated on an outpatient basis (c) patients with dependence on other substances except nicotine and caffeine (d) patients with mental subnormality or any condition interfering with the issue of consent except withdrawal delirium. Approval for the study was taken from the Institutional Ethics Committee.

ASSESSMENT: A semi structured questionnaire was used to collect the following data from the patients, post admission and informed consent (a) Socio demographic details (b) Clinical details.

The ICD-10 (DCR) WHO (1993) was used to determine the diagnosis of alcohol dependence. ${ }^{3}$ All patients meeting the criteria for dependence were included in the study.

Clinical Institute Withdrawal Assessment for Alcohol-revised (CIWA-Ar) was used to rate the withdrawal syndrome.4,5 It has a maximum score of sixty-seven and score of fifteen or more indicates increased risk of confusion and seizure. Subsequently CIWA-Ar was administered at eighthour intervals till the score touched zero regardless of discontinuation of pharmacotherapy around the fifth day. ${ }^{6,7}$ Delirium Rating Scale (DRS) was used to assess delirium. ${ }^{8}$ This is a 10 -point observer rated scale, which evaluates a variety of symptoms seen in delirium. Further a detailed clinical evaluation of the patients was done and patients were investigated and a complete blood count, differential count, haemoglobin, urine investigations, blood sugar, liver and renal function tests were performed. Sonology and other special indications were done if deemed necessary. Physical co morbidities were confirmed via consultation-liaison.

The sample was randomised into two groups and one group was put on a standardized fixed dose regimen of chlordiazepoxide and the other on a standardized regime of lorazepam. Fixed dose regimens were followed and dosage was based on recommendations in studies were dose of chlordiazepoxide ranged from 75-200 $\mathrm{mg}$ and lorazepam from 4-8 mg. The regime also included a vitamin B1 preparation and multivitamin capsules and an injection preparation of B1, B6, and B12. Patients in need for any other medication for co morbidities were excluded from the study.

STATISTICAL ANALYSES: The data were analysed and results were recorded as means and proportions. Statistical tests like t test, chi-square test and correlation analysis were done to analyse the different variables. The comparison of the groups based on continuous data where equal variance is assumed, was done using the independent sample t test. The strength of the association between two continuous variables e.g. severity of withdrawal and socio demographic and clinical variables and rate of fall of CIWA-Ar with these variables was analysed using Pearson's product moment correlation coefficient (Pearson's $r$ ). A stepwise logistic regression was also carried out. The analysis of statistical data was done using SPSS 15.0 program for Windows (Statistical Package for the Social Services)

RESULTS: A total of 103 patients $(n=103)$ were made part of the study. Post randomisation, the chlordiazepoxide sample had 42 patients (40.8\%) while 61 patients (59.2\%) received lorazepam, 


\section{ORIGINAL ARTICLE}

the disparity being due to the fact that 10 patients to be included in the chlordiazepoxide group were given lorazepam due to liver disease corroborated by LFT abnormalities.

The mean age of the sample was 43.4years and their distribution among the chlordiazepoxide and lorazepam group was 41.8 y vs. 44.6 y. The sample consisted mostly of people with low socio economic status (70.9\%) and the rest belonged to middle socioeconomic status (29.1\%), this is in keeping with the socio economic pattern of the population attending the hospital. With respect to marital status, 88 (85.4\%) were married, 9 (8.7\%) were unmarried; and 6 (5.8\%) were divorced or separated. Average age of onset of alcohol use was 18.1 years (18.4y vs. 17.9y). The average years of dependent use was found to be 16.4 years (13.3y vs.18.4y). The average daily alcohol consumption across different types of alcohol across the sample estimated for the last 30 days using a quantity frequency method and expressed as grams of ethanol is $237.9 \mathrm{~g}(195.3 \mathrm{~g}$ vs. $267.4 \mathrm{~g})$. Of the patients included 66 (64.1\%) had undergone previous detoxification treatment, and physical illness was the reason for current admission in 87 cases $(84.5 \%)$.

Alcoholic liver disease based on laboratory and sonographic evidence was seen in 49 cases (47.6\%). Of the sample 23 patients presented with delirium (22.3\%) and 21 patients (20.4\%) developed withdrawal seizures.

The comparability of the 2 groups with regards to factors like marital status, socioeconomic status, previous detoxification, family history of alcoholism, suicide and psychiatric illness were examined, the clinical factors like delirium, withdrawal seizures, and alcoholic liver disease were also studied. The two groups differed significantly with respect to marital status; family history of alcoholism and mental illness and also withdrawal seizures and delirium rates. This difference was due to the fact that all patients with liver disease were given lorazepam. The two groups were also compared based on age, age of onset of use, years of dependent use, and initial CIWA-Ar score. The two groups showed significant differences on parameters associated with alcoholic liver disease like age, years of dependent use and initial CIWA-Ar score and no significant difference with respect to other variables was seen.

The percentage improvement in CIWA- Ar after 48 hours of initiation of treatment was correlated with the different variables, to examine their role in the process of withdrawal, and whether these factors lead to a faster withdrawal or rapid decrease of the symptoms of withdrawal. The rate was also correlated with the initial CIWA-Ar score to see whether severity of withdrawal had a bearing on the rate of withdrawal. There was a significant correlation between the years of dependent use $(p=0.04)$, CIWA-Ar admission score $(p<0.001)$, SGOT and SGPT levels $(p<0.001)$ with initial improvement in CIWA-Ar score and the other socio demographic, clinical factors did not show any significant correlation. (Table 1) A subsequent linear regression showed that these factors did not contribute significantly to the improvement in CIWA-Ar score and most of the improvement was due to the drug given.

The percentage improvement in CIWA- Ar after 48 hours of initiation of treatment expressed in percentage showed that patients given chlordiazepoxide $(n=42)$ had a mean rate of fall of $57.4 \%($ S.D. $=5.9)$ and the lorazepam group ( $n=61$ ) showed a fall of $71.3 \%$ (S.D. $=4.7)$. The difference in the initial improvement on analysis using independent sample t-test, shows that the difference is statistically significant $(\mathrm{P}<0.001)$. The total duration of withdrawal calculated using the time it takes for the CIWA-Ar score to become zero, shows that chlordiazepoxide has a mean duration of 6.9days (S.D. =1.1) while lorazepam has a mean of 5.7 (S.D. =0.8), and this is statistically significant 


\section{ORIGINAL ARTICLE}

$(\mathrm{P}<0.001)$. The comparative results are summarised below. Thus lorazepam shows a statistically significant superiority to chlordiazepoxide both in influencing the rate of withdrawal and in shortening its duration. (Table 1and 2)

The other factors that had a significant correlation with improvement of withdrawal score were years of dependent use, amount of alcohol consumed per day, CIWA-Ar admission score and the SGOT and SGPT levels. (Table 1) A stepwise regression analysis was conducted taking these factors and the role of the drug and it was seen that $63 \%$ variance in initial improvement of CIWAAR score was due to the effect of the drug given. Therefore lorazepam was found to be significantly better than chlordiazepoxide in improving withdrawal symptoms.

DISCUSSION: The comparative rates of improvement in withdrawal symptoms, and the total duration of withdrawal symptoms, were related in a significant manner to the benzodiazepine given. With lorazepam there was a significant percentage improvement of CIWA-Ar score within 48 hours of instituting therapy (71.3\% vs. 57.4\%; p<0.001). The total duration of withdrawal was also significantly lower $(5.7 \mathrm{~d}$ vs. $6.9 \mathrm{~d} ; \mathrm{p}<0.001)$ a finding that is corroborated by a stepwise linear regression. There is evidence that lorazepam was as effective as chlordiazepoxide in reducing the symptoms of acute alcohol withdrawal. ${ }^{9}$ This study however suggests that lorazepam is superior. This finding also contradicts the observation that a drug with a longer half-life would provide smoother withdrawal. ${ }^{10}$ So also alcoholic liver disease is associated with severe withdrawal and certain factors are associated with liver disease, and when these are seen, it may be better to start the person on lorazepam. Therefore lorazepam is a safer and more effective drug than chlordiazepoxide. However further analysis is needed to confirm this finding, especially considering the small sample size of this study, and the observation that well-controlled comparison studies should be performed before recommending the routine use of lorazepam for treating alcohol withdrawal syndrome. ${ }^{11}$

The study though restricted by a smaller sample size and lack of double blinding suggests superiority of lorazepam. The short mean duration of withdrawal with both drugs shows that only a short duration of benzodiazepine therapy is needed during the withdrawal phase. Further it outlines the need for accurate assessment of withdrawal with a standardised instrument like CIWA-Ar, to identify those at increased risk, to institute appropriate treatment, and to minimise the overuse of benzodiazepines

TABLE 1: Factors Correlating with Initial CIWA-Ar Improvement

\begin{tabular}{|l|l|l|l|}
\hline & Pearson Correlation & Sig. (2-tailed) & $\mathrm{N}$ \\
\hline Age & 0.17 & $0.08^{*}$ & 103 \\
\hline Age of Onset & -0.13 & 0.19 & 103 \\
\hline Years of Dependent use & 0.281 & $0.004^{*}$ & 103 \\
\hline Amount of alcohol & 0.17 & 0.09 & 103 \\
\hline CIWA-Ar initial score & 0.26 & $0.01^{*}$ & 103 \\
\hline External Pressure & 0.04 & 0.66 & 103 \\
\hline SGOT & 0.42 & $<0.001^{*}$ & 103 \\
\hline SGPT & 0.38 & $<0.001^{*}$ & 103 \\
\hline
\end{tabular}


Table 2: Role of chlordiazepoxide and lorazepam in withdrawal

\begin{tabular}{|c|c|c|c|c|c|c|}
\hline & Drug & Mean & Std. Deviation & $\mathrm{T}$ & d. f. & Sig. \\
\hline \multirow{2}{*}{ CIWA Admission } & CHLORDIAZEPOXIDE & 17.7143 & 7.7846 & \multirow[t]{2}{*}{-3.25} & \multirow[t]{2}{*}{101} & \multirow[t]{2}{*}{$0.002^{*}$} \\
\hline & LORAZEPAM & 23.3934 & 9.3154 & & & \\
\hline \multirow{2}{*}{$\begin{array}{l}\text { Improvement of CIWA- } \\
\mathrm{Ar}\end{array}$} & CHLORDIAZEPOXIDE & 57.4214 & 5.8659 & \multirow{2}{*}{$\begin{array}{l}- \\
13.23\end{array}$} & \multirow[t]{2}{*}{101} & \multirow[t]{2}{*}{$<0.001^{*}$} \\
\hline & LORAZEPAM & 71.2767 & 4.7309 & & & \\
\hline \multirow{2}{*}{ TDW } & CHLORDIAZEPOXIDE & 6.9762 & 1.0930 & \multirow[t]{2}{*}{6.65} & \multirow[t]{2}{*}{101} & \multirow[t]{2}{*}{$<0.001^{*}$} \\
\hline & LORAZEPAM & 5.7049 & .8435 & & & \\
\hline
\end{tabular}

TDW: Total Duration of Withdrawal

\section{REFERENCES:}

1. Bayard M, McIntyre J, Hill KR, Woodside J Jr. Alcohol withdrawal syndrome, American Family Physician, Mar 15;69(6):1443-50.

2. Hoey LL, Nahum A, Vance-Bryan K. A retrospective review and assessment of benzodiazepines in the treatment of alcohol withdrawal in hospitalized patients, Pharmacotherapy, $14: 5,572-8$

3. ICD-10 classification of mental and behavioural disorders: Diagnostic Criteria for Research (1993), World Health Organisation.

4. Sullivan JT, Sykora K, Schneiderman J, Naranjo CA, Sellers EM. Assessment of alcohol withdrawal: The Revised Clinical Institute Withdrawal assessment for alcohol Scale (CIWAAr), British Journal of Addiction, 84,175-180.

5. Foy A, March S, Drinkwater V. Use of an objective clinical scale in the assessment and management of alcohol withdrawal in a large general hospital, Alcoholism: Clinical and Experimental Research, 12,360-364.

6. Kraemer KL, Mayo-Smith MF, Calkins DR. Independent clinical correlates of severe alcohol withdrawal, Substance Abuse, 24:4,197-209.

7. Saitz R, O'Malley SS. Pharmacotherapies for alcohol abuse: Withdrawal and Treatment, Medical Clinics of North America, 81:4, 881-907.

8. Trzepacz PT, Baker RW, Greenhouse J. A symptom rating scale for delirium, Psychiatry Res, 23:1, 89-97

9. Solomon J, Rouck LA, Koepke HH. Double-blind comparison of lorazepam and chlordiazepoxide in the treatment of the acute alcohol abstinence syndrome, Clin Ther, 6:1, 52-58.

10. MacKinnon GL, Parker WA, (1982) Benzodiazepine withdrawal syndrome: a literature review and evaluation, Am J Drug Alcohol Abuse, 9:1, 19-33.

11. Bird RD, Makela EH. Alcohol withdrawal: what is the benzodiazepine of choice? , Ann Pharmacother, 28:1, 67-71. 